From the University of Rochester Medical Center, Rochester, NY; and National Cancer Institute, National Institutes of Health, Rockville, MD.

Submitted April 5, 2010; accepted August 26, 2010; published online ahead of print at www.jco.org on October 25, 2010.

Presented in part at the 46th Annual Meeting of the American Society of Clinical Oncology, June 4-8, 2010, Chicago, IL.

Authors' disclosures of potential conflicts of interest and author contributions are found at the end of this article.

Corresponding author: Michael T. Milano, MD, PhD, Department of Radiation Oncology, University of Rochester Medical Center, 601 Elmwood Ave, Box 647, Rochester, NY 14642; e-mail: michael_milano@urmc.rochester.edu.

(C) 2010 by American Society of Clinical Oncology

0732-183X/10/2834-5088/\$20.00

DOI: $10.1200 / J C O .2010 .29 .5683$

\title{
Long-Term Survival Among Patients With Hodgkin's Lymphoma Who Developed Breast Cancer: A Population-Based Study
}

Michael T. Milano, Huilin Li, Mitchell H. Gail, Louis S. Constine, and Lois B. Travis

\section{$\begin{array}{llllllll}\text { A } & \text { B } & \text { S } & \text { T } & \text { R } & \text { A } & \text { C } & \text { T }\end{array}$}

\section{Purpose}

The increased risk of breast cancer $(\mathrm{BC})$ among women receiving chest radiotherapy for Hodgkin's lymphoma $(\mathrm{HL})$ is well-established. However, there are no large population-based studies that describe overall survival (OS) and cause-specific survival (CSS) compared with women with first primary BC.

\section{Methods}

For 298 HL survivors who developed BC (HL-BC group) and 405,223 women with a first or only BC (BC-1 group), actuarial OS and CSS were compared, accounting for age, BC stage, hormone receptor status, sociodemographic status, radiation for $\mathrm{HL}$, and other variables. All patients were derived from the population-based Surveillance, Epidemiology, and End Results program.

\section{Results}

OS among patients with $\mathrm{HL}-\mathrm{BC}$ was significantly inferior that of to patients with BC-1: 15-year OS was $48 \%$ versus 69\% $(P<.0001)$ for localized BC, and $33 \%$ versus $43 \%(P<.0001)$ for regional/distant $\mathrm{BC}$. Patients with $\mathrm{HL}-\mathrm{BC}$ had a significantly increased seven-fold risk $(P<.0001)$ of death from other cancers (ie, not $\mathrm{HL}$ or $\mathrm{BC}$ ) compared with patients with $\mathrm{BC}-1$. Mortality from heart disease among patients with $\mathrm{HL}-\mathrm{BC}$ with either localized or regional/distant disease was also significantly increased (hazard ratio $=2.22, P=.04$; and hazard ratio $=4.28, P=.02$, respectively) compared with patients with BC-1. Although 10-year BC-CSS was similar for patients with HL-BC and $\mathrm{BC}-1$ with regional/distant disease, it was inferior for patients with localized BC $(82 \% \vee 88 \%$, respectively; $P=.002$ ).

\section{Conclusion}

Women with $\mathrm{HL}$ may survive a subsequent diagnosis of $\mathrm{BC}$, only to experience significant excesses of death from other primary cancers and cardiac disease. Greater awareness of screening for cardiac disease and subsequent primary cancers in patients with HL-BC is warranted.

\section{J Clin Oncol 28:5088-5096. (c) 2010 by American Society of Clinical Oncology}

\section{INTRODUCTION}

Hodgkin's lymphoma (HL) is a highly curable disease, ${ }^{1}$ though the excellent life expectancy is offset by late effects of successful radiotherapy and chemotherapy, particularly the development of second malignancies. ${ }^{2-5}$ Fifteen to 30 years after therapy, cumulative mortality due to all second primary cancers exceeds deaths resulting from HL. ${ }^{3,6-8}$ Breast cancer (BC) accounts for the largest absolute risk of second cancers among female survivors of $\mathrm{HL}{ }^{2}$ with increased risks documented in numerous reports. ${ }^{2,4,5,9-16}$ For women treated for HL at age 25 years with chest doses of $\geq 40 \mathrm{~Gy}$, without alkylating agents, Travis et al ${ }^{12}$ estimated that the cumulative risk of BC by age 55 years is almost one in three. Despite these sobering statistics, few studies have addressed survival after BC diagnosis in women with HL. ${ }^{9,17-22}$ Most investigations consist of singleinstitution series, ${ }^{17,18,20,21}$ based on relatively small numbers of patients (range, 25 to 65 patients), followed-up for a median of less than 10 years. ${ }^{17-20}$ Although several investigations analyzed patient survival by BC stage, ${ }^{17-19}$ no studies have simultaneously controlled for the effects of age, hormone receptor status, year of $\mathrm{BC}$ diagnosis, and radiotherapy for HL. The actuarial calculation of other causes of death, from either heart disease, which is known to be significantly increased after radiotherapy for $\mathrm{HL},{ }^{23,24}$ or from other cancers, has 
also not been taken into account, although several reports addressed overall survival (OS), ${ }^{9,20-22}$ disease-free survival, ${ }^{17,20}$ and/or disease-specific survival. ${ }^{18,19,21}$

Within a cohort of 9,948 women with a first primary HL reported to population-based cancer registries comprising the Surveillance, Epidemiology, and End Results (SEER) program, we identified patients with a new primary BC diagnosis after HL (HL-BC group). We hypothesized that OS and BC cause-specific survival (CSS) of these patients would be worse than for women in the SEER database with a first or only primary BC diagnosis (BC-1 group) and would be impacted by sociodemographic status and race. We compared OS, BCCSS, CSS from other cancers, and heart disease CSS between groups. Patient-related, treatment-associated, and cancer-related variables were also analyzed to assess their effect on survival outcomes.

\section{METHODS}

\section{Patient Database}

From the US population-based SEER 9 (1973 to 2006) limited-use database, ${ }^{25} 317 \mathrm{HL}$ survivors were registered with an incident new primary BC. Fourteen were not actively followed-up by the SEER program, and in five others, BC stage was unavailable; the remaining 298 patients were included in our analyses. For the BC-1 comparison group, 405,223 women were analyzed. For both groups, women with unstaged $\mathrm{BC}$ or ductal carcinoma in situ were excluded, as were "death certificate only," "autopsy only," and cases without active follow-up. Women who developed additional ipsilateral or contralateral BCs after initial BC diagnosis were not excluded.

Of the 298 patients with HL-BC, 18 patients developed a second cancer before developing BC, including cervix $(n=7)$, thyroid $(n=5)$, vulva $(n=1)$, uterus $(\mathrm{n}=1)$, lip $(\mathrm{n}=1)$, lung $(\mathrm{n}=1)$, bladder $(\mathrm{n}=1)$, and non-Hodgkin's lymphoma (NHL, $\mathrm{n}=1$ ). At last follow-up, 12 of these 18 patients were alive, and six had died: three as a result of $\mathrm{BC}$, one as a result of "miscellaneous cancer," one as a result of "other cause of death," and one as a result of sepsis.

HL stage was determined from SEER extent of disease fields (see Appendix, online only). $\mathrm{BC}$ was staged using the "SEER historic stage A" variable, categorized as limited (ie, confined to breast tissue, excluding T4 tumors), regional (ie, lymph node involvement and/or locally advanced tumors), or distant (ie, metastatic). Information about radiation fields, radiation dose, chemotherapy, or hormonal therapy is not available in the SEER database, though radiotherapy for HL usually includes thoracic radiation.

\section{Statistical Analysis}

Actuarial OS and CSS were calculated using the Kaplan-Meier method. "Any other cancer" CSS reflects death from any cancer other than BC or HL. Survival times were measured from date of $\mathrm{BC}$ diagnosis until date of death or last follow-up. For the BC-1 group, actuarial survival was calculated for randomly selected subsets of patients, matched to the HL-BC group for sociodemographic status, age, and year of $\mathrm{BC}$ diagnosis (described in the Appendix, online only). Cox regression was used to compare survival outcomes, controlling for covariates. All analyses were conducted using SAS 9.1.3 software (SAS Institute, Cary, NC). Kaplan-Meier curves were prepared using R 2.7.0. ${ }^{26}$

\section{RESULTS}

\section{Patient and Tumor Characteristics}

Patient and tumor characteristics at time of HL diagnosis, grouped by stage of subsequent BC, are outlined in Table 1. Seventythree percent of patients received HL treatment before age 40 years, the time of greatest radiocarcinogenicity of the breast. ${ }^{16,27}$ Eighty percent of women younger than 40 years and $73 \%$ of all women
Table 1. Patient and Tumor Characteristics at Time of HL Diagnosis Among 298 Women Who Developed a Second Primary Breast Cancer

\begin{tabular}{|c|c|c|c|c|c|c|c|c|c|}
\hline \multirow[b]{3}{*}{ Characteristic } & \multicolumn{9}{|c|}{ Second Primary Breast Cancer } \\
\hline & \multicolumn{2}{|c|}{ All } & \multicolumn{2}{|c|}{ Localized } & \multicolumn{2}{|c|}{ Regional } & \multicolumn{2}{|c|}{ Distant } & \multirow[b]{2}{*}{$P^{*}$} \\
\hline & No. & $\%$ & No. & $\%$ & No. & $\%$ & No. & $\%$ & \\
\hline Total No. of patients & $298+$ & & 187 & & 90 & & 21 & & \\
\hline $\begin{array}{l}\text { Age at } \mathrm{HL} \text { diagnosis, } \\
\text { years }\end{array}$ & & & & & & & & & .016 \\
\hline$<10-19$ & 66 & 22 & 33 & 18 & 28 & 31 & 5 & 24 & \\
\hline 20-39 & 152 & 51 & 96 & 51 & 46 & 51 & 10 & 48 & \\
\hline $40-59$ & 48 & 16 & 34 & 18 & 10 & 11 & 4 & 19 & \\
\hline$\geq 60$ & 32 & 11 & 24 & 13 & 6 & 7 & 2 & 10 & \\
\hline Race & & & & & & & & & .36 \\
\hline White & 270 & 91 & 173 & 93 & 80 & 89 & 17 & 81 & \\
\hline Black & 24 & 8 & 12 & 6 & 9 & 10 & 3 & 14 & \\
\hline Other & 4 & 1 & 2 & 1 & 1 & 1 & 1 & 5 & \\
\hline Year of $\mathrm{HL}$ diagnosis & & & & & & & & & .06 \\
\hline 1973-1979 & 100 & 34 & 67 & 36 & 30 & 33 & 3 & 14 & \\
\hline 1980-1989 & 145 & 49 & 87 & 47 & 48 & 53 & 10 & 48 & \\
\hline 1990-1999 & 49 & 16 & 32 & 17 & 20 & 11 & 7 & 33 & \\
\hline 2000-2006 & 4 & 1 & 1 & 1 & 2 & 2 & 1 & 5 & \\
\hline Subtype of $\mathrm{HL}$ & & & & & & & & & .35 \\
\hline Nodular sclerosis & 213 & 71 & 132 & 71 & 64 & 71 & 17 & 81 & \\
\hline Mixed cellularity & 43 & 14 & 32 & 17 & 8 & 9 & 3 & 14 & \\
\hline Lymphocyte depleted & 7 & 2 & 4 & 2 & 2 & 2 & 1 & 5 & \\
\hline $\begin{array}{l}\text { Nodular lymphocyte } \\
\text { predominant or } \\
\text { lymphocyte rich }\end{array}$ & 10 & 3 & 7 & 4 & 3 & 3 & 0 & & \\
\hline Classic, NOS & 25 & 8 & 12 & 6 & 13 & 14 & 0 & & \\
\hline Stage of HL $\ddagger$ & & & & & & & & & .50 \\
\hline 1 & 85 & 29 & 56 & 30 & 23 & 26 & 6 & 29 & \\
\hline$\|$ & 104 & 35 & 64 & 34 & 36 & 30 & 5 & 24 & \\
\hline III-IV & 94 & 31 & 57 & 30 & 27 & 20 & 10 & 48 & \\
\hline Unknown & 14 & 5 & 10 & 5 & 4 & 4 & 0 & & \\
\hline Radiotherapy for $\mathrm{HL}$ & & & & & & & & & .74 \\
\hline Yes & 217 & 73 & 136 & 73 & 67 & 74 & 14 & 67 & \\
\hline No & 75 & 25 & 46 & 25 & 22 & 24 & 7 & 33 & \\
\hline Unknown & 6 & 2 & 5 & 3 & 1 & 1 & 0 & & \\
\hline
\end{tabular}

Abbreviations: HL, Hodgkin's lymphoma; NOS, not otherwise specified. * $P$ values represent $\chi^{2}$ test comparing distribution of the specific variables between the localized, regional, and distant groups.

†For an additional five HL survivors who developed breast cancer, stage was not provided in the Surveillance, Epidemiology, and End Results database. $\neq$ Refer to the online-only Appendix for an explanation of the assignment of $\mathrm{HL}$ stage. In women assigned more than one possible HL stage, the higher stage is depicted in this table.

received radiation for HL. More than $80 \%$ were diagnosed with HL before 1990.

Table 2 outlines clinicopathologic features of patients with HL-BC and BC-1 at time of BC diagnosis. For patients with HL-BC, 187 (63\%), 90 (30\%), and 21 (7\%) had localized, regional, and distant $\mathrm{BC}$, respectively, with comparable percentages $(60 \%, 34 \%$, and $6 \%$, respectively) for first primary BCs. Among women with HL-BC, 67\% diagnosed before 1996 had localized disease versus 61\% diagnosed from 1996 to 2006 ( $P=.33$ ). Because only 21 patients with HL-BC had distant disease (Table 1), regional and distant subgroups were combined for subsequent analyses. Median latency between diagnoses of $\mathrm{HL}$ and $\mathrm{BC}$ was 15.2 years (range, 0.6 to 33.3 years) and 15.3 years (range, 0.3 to 32.0 years), respectively, for localized and regional/ distant BC. Patients with HL-BC were significantly $(P<.0002)$ 


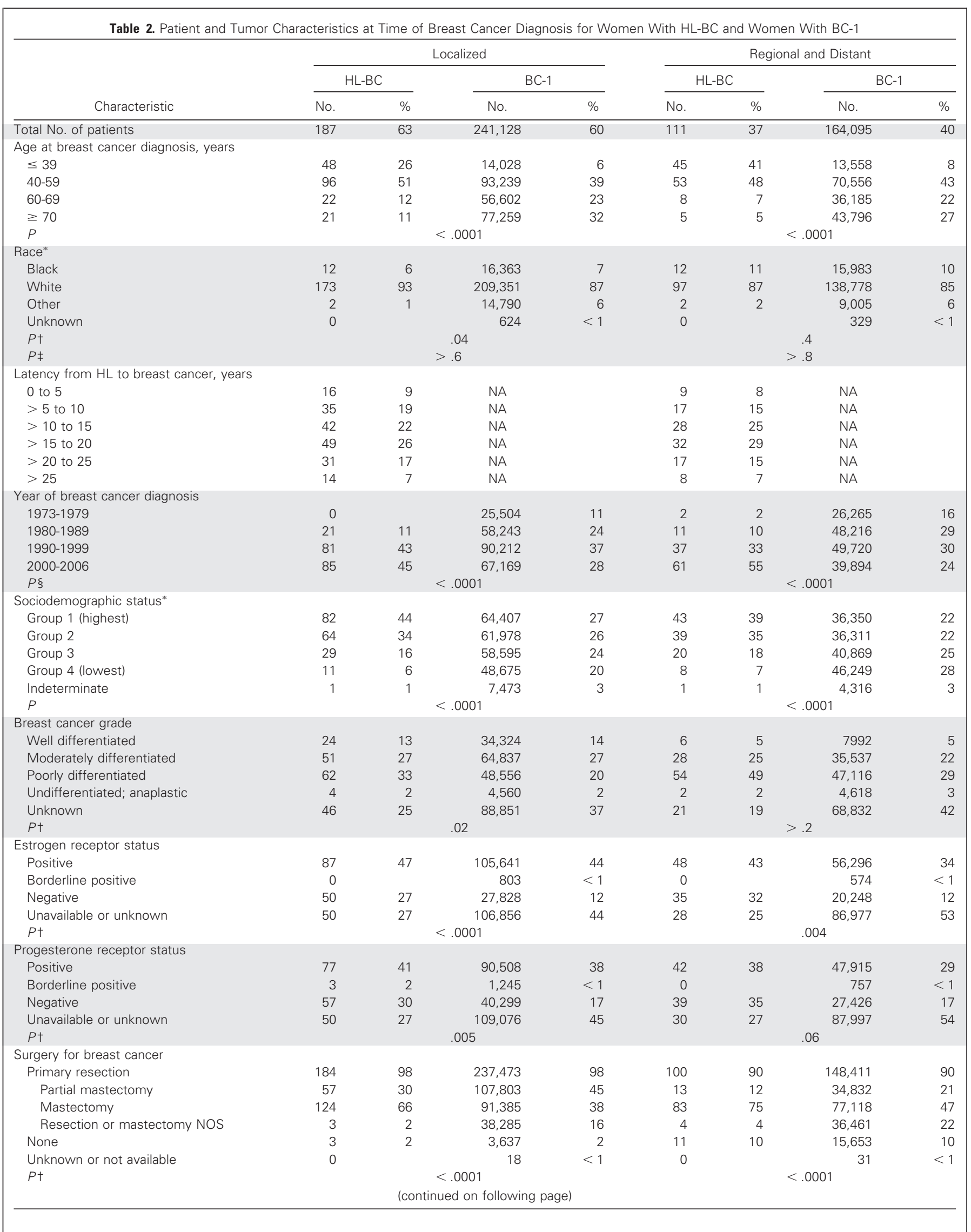


Table 2. Patient and Tumor Characteristics at Time of Breast Cancer Diagnosis for Women With HL-BC and Women With BC-1 (continued)

\begin{tabular}{|c|c|c|c|c|c|c|c|c|}
\hline \multirow[b]{3}{*}{ Characteristic } & \multicolumn{4}{|c|}{ Localized } & \multicolumn{4}{|c|}{ Regional and Distant } \\
\hline & \multicolumn{2}{|c|}{ HL-BC } & \multicolumn{2}{|c|}{$\mathrm{BC}-1$} & \multicolumn{2}{|c|}{ HL-BC } & \multicolumn{2}{|c|}{$\mathrm{BC}-1$} \\
\hline & No. & $\%$ & No. & $\%$ & No. & $\%$ & No. & $\%$ \\
\hline \multicolumn{9}{|l|}{ Radiation for breast cancer } \\
\hline Radiation delivered & 36 & 19 & 91,493 & 38 & 16 & 14 & 61,696 & 38 \\
\hline External beam & 33 & & 87,971 & & 15 & & 60,173 & \\
\hline Radioactive implants & 3 & & 960 & & 0 & & 120 & \\
\hline Combination beam + implants & 0 & & 1,731 & & 1 & & 774 & \\
\hline Radiation NOS & 0 & & 831 & & 0 & & 629 & \\
\hline None & 146 & 78 & 144,755 & 60 & 92 & 83 & 96,725 & 59 \\
\hline Refused & 0 & & 1,442 & $<1$ & 0 & & 1,051 & 1 \\
\hline Unknown & 5 & 3 & 3,438 & 1 & 3 & 3 & 4,623 & 3 \\
\hline$P+$ & \multicolumn{4}{|c|}{$<.0001$} & \multicolumn{4}{|c|}{$<.0001$} \\
\hline
\end{tabular}

NOTE. $P$ values represent $\chi^{2}$ test comparing the distribution between $\mathrm{HL}-\mathrm{BC}$ and $\mathrm{BC}-1$ patients for the indicated variable.

Abbreviations: HL-BC, breast cancer after Hodgkin's lymphoma; BC-1, first or only primary breast cancer; NA, not applicable; NOS, not otherwise specified.

*At the time of breast cancer diagnosis for each patient, the proportion of adults age $\geq 25$ years within the same county of residence with less than a high-school education was determined from census data, as a surrogate for sociodemographic status. ${ }^{28}$ The table depicts quartiles of sociodemographic status. For those patients with missing information, sociodemographic status is considered indeterminate.

tRepresents $\chi^{2}$ test omitting patients with unknown or unavailable variables.

\#Represents $\chi^{2}$ test comparing only the distribution of black and white patients between the HL-BC $v$ BC- 1 groups. The significant difference $(P=.04)$ in the first

$\chi^{2}$ test reflects a greater percentage of patients with other race (American Indian/Alaska Native, Asian/Pacific Islander) in the BC-1 group.

§Refer to text. The more recent calendar year intervals of breast cancer diagnosis observed for the HL-BC patients is expected, given that Hodgkin's lymphoma diagnosed before 1973 would not have been reported to the SEER registries, in conjunction with the latency period of about 10 years for the development of radiation-induced breast cancer.

younger than patients with BC-1 (median age, 45 and 61 years, respectively) and had a higher sociodemographic status (defined in Table 2). ${ }^{28}$ Only 28 patients with HL-BC were nonwhite, consistent with the more common occurrence of HL in whites. Exclusion of these patients from subsequent analyses did not impact results. A greater proportion of BC-1 patients had unknown tumor grade, and unknown estrogen receptor (ER) and progesterone receptor (PR) status. The latter finding reflects the fact that most (89\%) patients with HL-BC were diagnosed after 1990, when SEER registries initiated collection of ER and PR data. Among patients with BC for whom hormonal status was reported, those with antecedent HL were significantly more likely to have ER-negative and PR-negative BC. Among patients with localized $\mathrm{BC}$ for whom grade was reported, those with antecedent HL were more likely to have poorly differentiated cancer $(P=.02)$.

There was no significant difference in tumor size distribution between patients with HL-BC and BC-1 with localized disease (data not shown). For localized BC, patients in the HL-BC group were more likely to have undergone a complete mastectomy than those in the BC-1 group $(66 \% v 38 \% ; P<.0001)$ and less likely to have undergone radiotherapy $(19 \% v 38 \% ; P<.0001)$. Similar findings were observed among patients with HL-BC with regional/distant BC. Among patients with HL-BC having localized BC, 35\% who underwent partial mastectomy did not receive breast radiation (70\% of these patients had received prior radiation for $\mathrm{HL}$ ), as compared with $22 \%$ of patients with $\mathrm{BC}-1(P=.015)$.

\section{Vital Status at Last Follow-Up and Cause of Death}

Table 3 outlines vital status at last follow-up and causes of death. Of patients with HL-BC, 55\%, 28\%, 10\%, and 7\% were followed up less than 5,5 to 9,10 to 14 , and $\geq 15$ years after $\mathrm{BC}$ diagnosis. Comparable numbers in the BC-1 cohort were $40 \%, 26 \%, 16 \%$, and $19 \%$, respectively. Of patients with HL-BC, 109 (37\%) were deceased, compared with 187,308 (46\%) of patients with BC-1. Of 58 women with $\mathrm{HL}-\mathrm{BC}$ having localized $\mathrm{BC}$ who died, comparable percentages died from BC (38\%) and other cancers (34\%), whereas no deaths were attributed to HL. Not surprisingly, of 51 deaths among women with HL-BC having regional/distant BC, most resulted from BC (59\%), similar to $64 \%$ of deaths in the BC-1 group.

For 28 patients with HL-BC who died of other cancers, median latency from HL to BC was 12.6 years (range, 0.6 to 27 years). Twenty-two had received radiation for $\mathrm{HL}$, diagnosed at age 13 to 75 years (median, 29 years). Causes of death were lung cancer $(\mathrm{n}=8)$, NHL $(\mathrm{n}=7)$, esophageal cancer $(\mathrm{n}=1)$, colon cancer $(\mathrm{n}=1)$, anal cancer $(\mathrm{n}=1)$, sarcoma $(\mathrm{n}=1)$, and "miscellaneous malignant cancer" $(n=9)$. Among the seven patients whose death was attributed to NHL, five patients died $\geq 5$ years after HL diagnosis ( $\geq 10$ years in four patients). Among the nine patients dying from miscellaneous malignant cancer, four patients had third primary cancers reported to the SEER program (metastatic carcinoid, metastatic lung cancer, sarcoma, and malignant meningioma) that could have accounted for their deaths. Among the 19 other patients for whom a specific cancer death was reported, 11 patients had a separate SEER case listing that matched the cause of death, whereas eight patients did not. Among these 11 patients, the median latency from diagnosis of HL to the third primary cancer was 18 years (range, 5.2 to 31 years). Subsequent survival for these 11 patients was poor (median, 7 months; range, 2 months to 5.2 years).

Eight patients with HL-BC ( $10 \%$ of 83 cancer-related deaths) and 4,595 patients with BC-1 (4\% of 106,208 cancer-related deaths) died as a result of lung/bronchial cancer; nine patients with HL-BC ( $11 \%$ of 83 cancer-related deaths) and 2,992 patients in the BC-1 group (3\% of 106,208 cancer-related deaths) died as a result of miscellaneous malignant cancers. These differences between the $\mathrm{HL}-\mathrm{BC}$ and $\mathrm{BC}-1$ groups in the rates of deaths from lung/bronchial 
Table 3. Vital Status at Last Follow-Up and Cause of Death: Women With HL-BC $(n=298)$ and Women With BC-1 ( $n=405,223)$

\begin{tabular}{|c|c|c|c|c|c|c|c|c|c|c|c|c|c|c|c|c|}
\hline \multirow[b]{3}{*}{ Vital Status } & \multicolumn{4}{|c|}{ Localized } & \multicolumn{4}{|c|}{ Regional } & \multicolumn{4}{|c|}{ Distant } & \multicolumn{4}{|c|}{ Regional/Distant } \\
\hline & \multicolumn{2}{|c|}{ HL-BC } & \multicolumn{2}{|c|}{$B C-1$} & \multicolumn{2}{|c|}{ HL-BC } & \multicolumn{2}{|c|}{$B C-1$} & \multicolumn{2}{|c|}{ HL-BC } & \multicolumn{2}{|c|}{$B C-1$} & \multicolumn{2}{|c|}{ HL-BC } & \multicolumn{2}{|c|}{$B C-1$} \\
\hline & No. & $\%$ & No. & $\%$ & No. & $\%$ & No. & $\%$ & No. & $\%$ & No. & $\%$ & No. & $\%$ & No. & $\%$ \\
\hline Alive & 129 & 69 & 151,892 & 63 & 54 & 60 & 62,246 & 45 & 6 & 29 & 3777 & 15 & 60 & 54 & 66,023 & 40 \\
\hline Deceased & 58 & 31 & 89,236 & 37 & 36 & 40 & 75,918 & 55 & 15 & 71 & 22,154 & 85 & 51 & 46 & 98,072 & 60 \\
\hline \multicolumn{17}{|l|}{ Cause of death* } \\
\hline Breast cancer & $22 \dagger$ & 38 & $24,989+$ & 28 & 20 & 56 & 44,670 & 59 & 10 & 67 & 18,234 & 82 & 30 & 59 & 62,904 & 64 \\
\hline Hodgkin's lymphoma & 0 & & 18 & $<1$ & 2 & 6 & 10 & $<1$ & 1 & 7 & 0 & & 3 & 6 & 10 & $<1$ \\
\hline Other cancer $\ddagger$ & 20 & 34 & 11,292 & 13 & 5 & 14 & 5,777 & 8 & 3 & 20 & 1,218 & 5 & 8 & 16 & 6,995 & 7 \\
\hline Heart disease & 8 & 14 & 20,957 & 23 & 3 & 8 & 10,443 & 14 & 0 & & 1,053 & 5 & 3 & 6 & 11,496 & 12 \\
\hline Other causes§ & 8 & 14 & 31,980 & 36 & 6 & 17 & 15,018 & 20 & 1 & 7 & 1,649 & 7 & 7 & 14 & 16,667 & 17 \\
\hline All & 187 & & 24,1128 & & 90 & & 138,164 & & 21 & & 25,931 & & 111 & & 164,095 & \\
\hline
\end{tabular}

Abbreviations: HL-BC, breast cancer after Hodgkin's lymphoma; BC-1, first or only primary breast cancer

*Percentages for cause of death represent percentage of all deaths.

tFor women with localized breast cancer who died as a result of breast cancer, 4 (18\%) of 22 HL-BC patients were registered in the Surveillance, Epidemiology, and End Results program as developing an incident contralateral breast cancer $v 1,956(8 \%)$ of 24,989 of $\mathrm{BC}-1$ patients $(P=.071)$.

¥Other cancer causes of death included lung $(n=8)$, esophagus $(n=1)$, colon $(n=1)$, anal $(n=1)$, non-Hodgkin's lymphoma $(n=5)$, sarcoma ( $n=1)$, and miscellaneous cancer $(n=3)$ among women with localized breast cancer and non-Hodgkin's lymphoma $(n=2)$ and miscellaneous cancer ( $n=6)$ among women with regional/distant breast cancer.

$\S$ For women with localized breast cancer, other causes included chronic liver disease $(n=1)$, chronic obstructive lung disease $(n=1)$, pneumonia or influenza $(n=1)$, other causes (not specified, $n=3$ ), and not available $(n=2)$. For women with regional/distant breast cancer, other causes included chronic liver disease $(n=1)$, hypertension without heart disease $(n=1)$, ill-defined condition $(n=1)$, infectious disease or sepsis $(n=2)$, and other causes $(n o t$ specified, $n=2)$.

cancer and miscellaneous cancers are consistent with the known increased risk of solid tumors among HL survivors (see Discussion).

\section{Survival of HL-BC Versus BC-1 Groups}

Table 4 and Figures 1A through $1 \mathrm{C}$ summarize the actuarial OS, BC-CSS, and other cancer CSS probabilities of patients with HL-BC and BC-1, matched for age, year of BC diagnosis, and sociodemographic status. Table 4 shows $P$ values and hazard ratios (HRs) derived from Cox models comparing survival. Adjusting for covariates, the HL-BC group had a significantly poorer OS. Patents in the HL-BC group having localized disease had a significantly two-fold increased mortality $(P<.0001)$ from either BC or heart disease as compared with patients in the $\mathrm{BC}-1$ group. Patients in the HL-BC group with regional/distant disease demonstrated a significantly increased fourfold relative mortality from heart disease, with a nonsignificant $30 \%$ excess risk of death resulting from BC. It is noteworthy that HL survivors with localized or regional/distant BC had approximately seven times the hazard of death from other cancers as compared with patients with BC-1. Patients with regional/distant disease had a 4.7fold increased risk of death from causes other than cancer or heart disease (refer to Table 3 footnote).

\section{Prognostic Factors Among HL Survivors With BC}

Table 5 shows results of univariate and multivariate analyses of prognostic factors that could potentially impact OS or BC-CSS in patients with HL-BC. For OS, age at BC diagnosis was inversely related to survival $(P=.0003$ and $P=.004$, respectively, for women with either localized or regional/distant BC). ER negativity was an adverse prognostic factor for OS among women with distant/regional BC. For women with localized BC, lower sociodemographic status was an adverse predictor $(P=.04)$ of BC-CSS. For women with regional/ distant $\mathrm{BC}$, radiation for $\mathrm{HL}(\mathrm{HR}=2.61 ; P=.03)$ and $\mathrm{ER}$ negativity $(\mathrm{HR}=3.07 ; P=.02)$ were significant adverse risk factors for $\mathrm{BC}-\mathrm{CSS}$.
No other tested variable was a statistically significant predictor for OS or CSS in the multivariate analyses.

\section{DISCUSSION}

An important new finding in our study, based on 298 patients with $\mathrm{HL}-\mathrm{BC}$ and 405,223 patients with BC-1, is the observation that $\mathrm{HL}$ survivors in the general population who develop $\mathrm{BC}$, as compared with women with a first or only BC, are at a significantly increased seven-fold risk of death from other cancers. Moreover, these patients experience significantly elevated two- to four-fold greater risks of cardiac death. For the first time, to our knowledge, the OS and CSS beyond 10 years, as well as the influence of several clinicopathologic factors (ie, sociodemographic status) on survival outcomes, are evaluated among patients with HL who develop BC. We show that lower sociodemographic status adversely affects both BC-CSS $(P=.04)$ and OS $(P=.09)$ among women who develop localized BC after HL.

Whereas the increased risk of new primary cancers in patients with HL is well-established, 2,6,7,22,29 few studies address the development of two or more cancers after $\mathrm{HL}^{2}$ or subsequent mortality due to these malignancies. ${ }^{6,7}$ To our knowledge, none have addressed cancer mortality among HL survivors who develop BC. In our study, deaths from third or high-order cancers occurred at sites for which significantly increased risks of second cancers have been reported., 2,4,22,29-31 including $\mathrm{NHL},{ }^{2,4,5,22,31,32}$ cancers of lung, ${ }^{2,4,22,29,31,33}$ esophagus, ${ }^{2,4,30,31}$ colon, ${ }^{2,4,22,30,31}$ anus, ${ }^{30}$ soft tissue, ${ }^{2,4,30,31}$ and brain. ${ }^{2,30}$

HL survivors have a significantly increased three- to 10 -fold risk of lung cancer, ${ }^{2,4,22,29,31,33}$ with associated risk factors including prior radiation, alkylating-agent chemotherapy (both with significant doseresponses), and smoking history. ${ }^{33,34}$ Virtually all lung cancers develop in HL survivors who smoke. ${ }^{33,34}$ Thoracic radiation and smoking history also significantly increase the risk of lung cancer after BC. ${ }^{35,36}$ Although it is possible that deaths resulting from metastatic 
Table 4. Comparison of Actuarial Survival Probabilities: Women With HL-BC $(n=298)$ Versus Women With BC-1 ( $n=405,223$ )

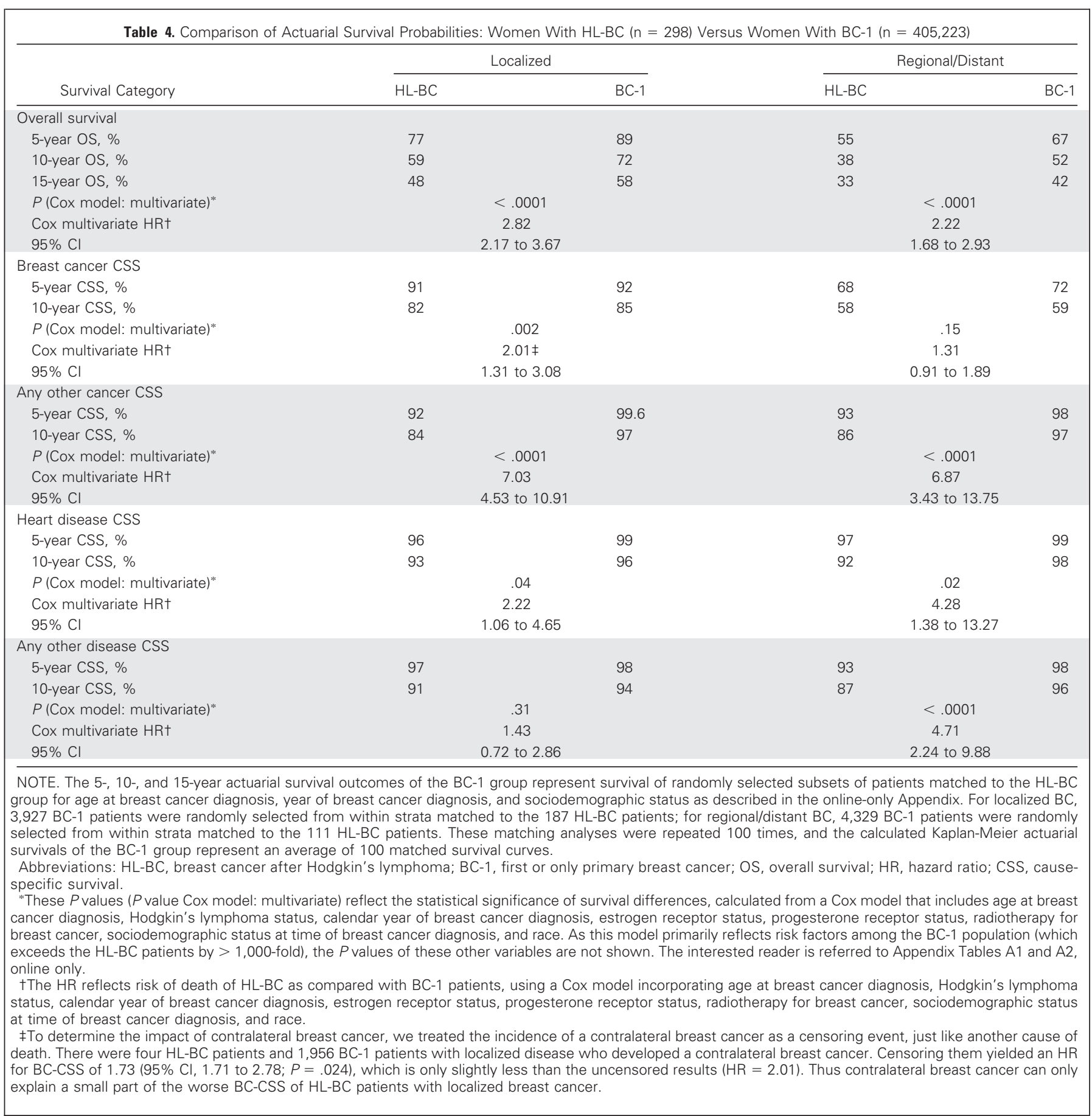

$\mathrm{BC}$ were erroneously ascribed to lung cancer for either patients with HL-BC or BC-1, the reported rate of such misclassification is low. ${ }^{37}$ Without independent histopathologic review, unequivocal classification of incident lung cancer after BC is not possible.

HL survivors have a significantly increased five-fold to more than 20 -fold risks of NHL, with excesses persisting for more than 15 years after HL. ${ }^{2,4,5,22,31,32}$ Although death attributed to NHL in our study could have represented misclassified HL, this type of error is less likely in patients with a lengthy interval between the two lymphoma diagnoses. Similarly, although women originally assigned a diagnosis of
HL could have actually had NHL, this type of misclassification occurs in only approximately $2 \%$ of patients. ${ }^{32}$

Using an actuarial comparison, patients in the HL-BC group had a significantly increased two- to four-fold risk of death from heart disease as compared with patients in the BC-1 group. Although patients with either $\mathrm{HL}^{23}$ or $\mathrm{BC}^{38}$ are susceptible to treatment-related cardiac toxicities (attributable to radiation and/or anthracycline chemotherapy), HL survivors tend to receive these exposures at a considerably younger age. Moreover, compared with patients with $\mathrm{BC}$, patients with $\mathrm{HL}$ are generally 


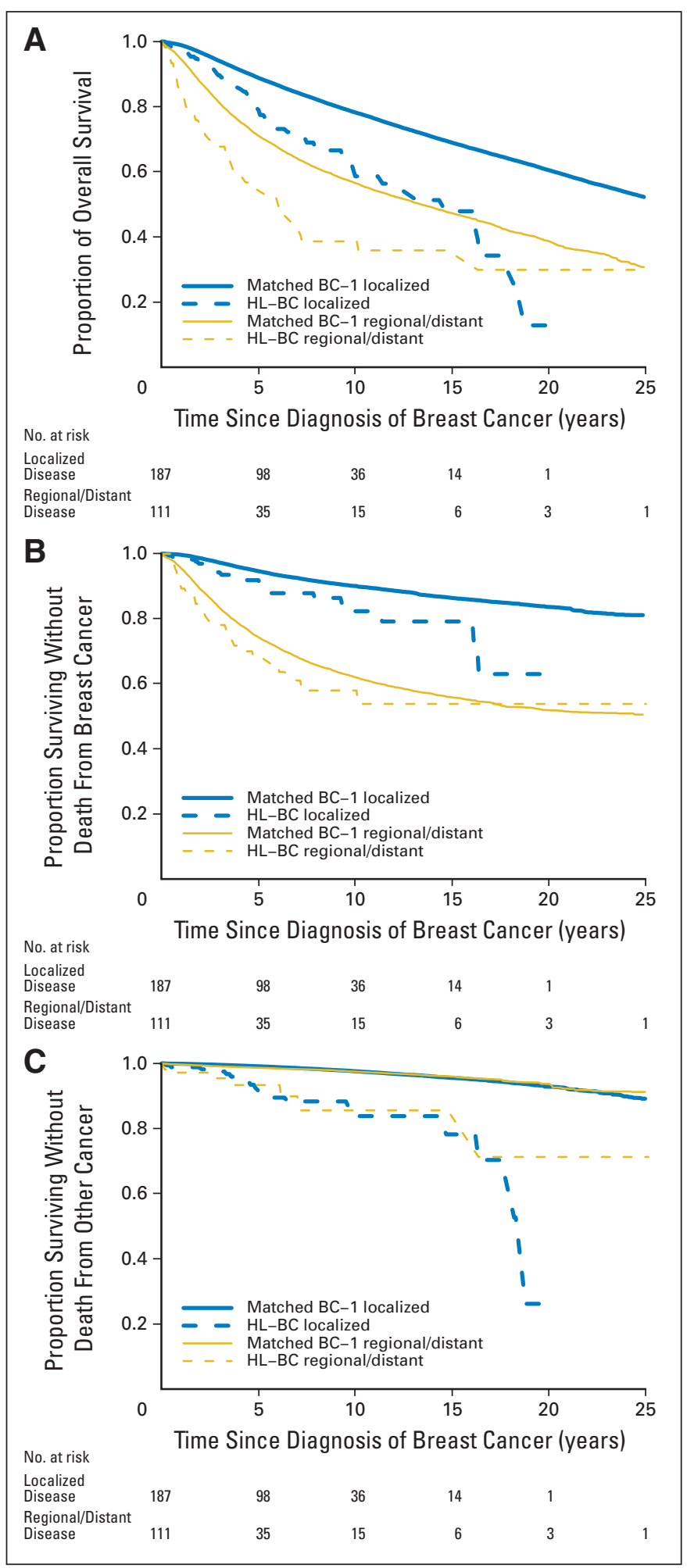

Fig 1. Comparison of (A) overall survival, (B) survival without death from breast cancer, and (C) survival without death from any other cancer (ie, excluding Hodgkin's lymphoma [HL] and breast cancer) for women with first primary breast cancer (BC-1, solid lines) and those with breast cancer after $\mathrm{HL}$ ( $\mathrm{HL}-\mathrm{BC}$, dashed lines). For the BC-1 group, actuarial survival was calculated for a subset and matched to the HL-BC group for sociodemographic status, age, and year of BC diagnosis (described in online-only Appendix and Table 4 footnotes). Estimates for localized breast cancer (BC-1 and $\mathrm{HL}-\mathrm{BC}$ ) are shown in blue, whereas those for regional/distant disease (BC-1 and $\mathrm{HL}-\mathrm{BC}$ ) are shown in gold. Number at risk indicates the number of patients with $\mathrm{HL}$ with new primary breast cancer at risk at the indicated time interval.

\begin{tabular}{|c|c|c|c|c|}
\hline \multirow[b]{2}{*}{ Variable } & \multicolumn{2}{|c|}{ Localized } & \multicolumn{2}{|c|}{ Regional/Distant } \\
\hline & $\begin{array}{l}\text { Overall } \\
\text { Survival }\end{array}$ & $\begin{array}{l}\text { BC Cause- } \\
\text { Specific } \\
\text { Survival }\end{array}$ & $\begin{array}{l}\text { Overall } \\
\text { Survival }\end{array}$ & $\begin{array}{c}\text { BC Cause- } \\
\text { Specific } \\
\text { Survival }\end{array}$ \\
\hline \multicolumn{5}{|l|}{ Univariate analysis, $P$} \\
\hline HL stage* & $.89-.99$ & $.74-.90$ & $.42-.94$ & $.51-.80$ \\
\hline Radiation for $\mathrm{HL}$ & .62 & .45 & .30 & .04 \\
\hline Latency of BC† & .48 & .88 & .22 & .24 \\
\hline Age at $\mathrm{BC}$ diagnosis ${ }^{\dagger}$ & $<.001$ & .19 & .005 & .52 \\
\hline Year of $B C$ diagnosis $\dagger$ & .10 & .40 & .90 & 27 \\
\hline Sociodemographic status $\dagger$ & .02 & .06 & .63 & .28 \\
\hline ER status $¥$ & .42 & .45 & .16 & .02 \\
\hline ER status§ & .86 & .42 & .06 & .02 \\
\hline PR status $¥$ & .88 & .38 & .15 & .04 \\
\hline PR status§ & 0.66 & .87 & .06 & .04 \\
\hline \multicolumn{5}{|l|}{ Multivariate analysis } \\
\hline Radiation for $\mathrm{HL}$ & ND & ND & ND & \\
\hline$P$ & & & & .03 \\
\hline HR & & & & 2.61 \\
\hline Age at $\mathrm{BC}$ diagnosis & & & & ND \\
\hline$P$ & .0003 & .14 & .004 & \\
\hline$H R$ & 1.04 & 0.980 & 1.03 & \\
\hline Year of $\mathrm{BC}$ diagnosis & & ND & ND & ND \\
\hline$P$ & .96 & & & \\
\hline $\mathrm{HR}$ & 1.00 & & & \\
\hline Sociodemographic status & & & ND & ND \\
\hline$P$ & .09 & .04 & & \\
\hline$H R$ & 1.05 & 1.08 & & \\
\hline ER negative & ND & ND & & \\
\hline$P$ & & & .05 & .02 \\
\hline$H R$ & & & 2.06 & 3.07 \\
\hline
\end{tabular}

NOTE. Variables with $P$ values $<.2$ in the univariate analyses were entered into the multivariate analysis. PR status was not entered into the multivariate model because of the small sample size and high correlation between ER status and PR status.

Abbreviations: HL, Hodgkin's lymphoma; BC breast cancer; ER, estrogen receptor; PR, progesterone receptor; ND, not done, because $P$ value was $>0.2$ with univariate analysis; HR, hazard ratio.

*As described in the online-only Appendix, $\mathrm{HL}$ stage was assigned in four different ways. The influence of stage was not statistically significant when the influence of stage was tested using any of these schema. The range of $P$ values is shown in the table.

†These variables were analyzed with a Cox model using the single variable of interest; all others were analyzed using the log-rank method.

fLog-rank test which reflects difference in survival between patients according to hormone receptor status (ie, positive $v$ negative $v$ borderline $\checkmark$ unknown).

$\S$ These analyses excluded patients for whom receptor status was either borderline or unknown.

given higher anthracycline doses and are irradiated to larger cardiac volumes, albeit at lower doses. ${ }^{39}$ The higher risk of cardiac death after HL is also noteworthy in view of the common use of mechlorethamine, vincristine, procarbazine, and prednisone chemotherapy during much of the study period (1970s to mid 1980s), after which doxorubicin, bleomycin, vinblastine, and dacarbazine (which includes cardiotoxic doxorubicin) became more widely used. ${ }^{40}$

Among women with localized $\mathrm{BC}$, patients in the HL-BC group had a significantly increased two-fold risk of death resulting from $\mathrm{BC}$ as compared with patients in the $\mathrm{BC}-1$ group, which may in part be 
due to an approximately two-fold (albeit nonsignificant) greater prevalence of contralateral BC (Table 3). However, contralateral BC explains only a small part of the inferior BC-CSS (Table 4), which may also reflect patient susceptibility or treatment-induced factors and/or limitations in treatment options for BC after HL. For example, HL survivors are less likely to receive anthracycline chemotherapy for $\mathrm{BC} .^{21}$ Moreover, our study and another investigation ${ }^{21}$ also showed that HL survivors are significantly less likely to receive breastconserving surgery or radiotherapy for BC than patients with BC-1. We also found that HL survivors who underwent breast-conserving surgery were less likely to receive radiation, most likely due to prior chest radiation for $\mathrm{HL}$.

BC after treatment for HL may also exhibit a different biology compared with de novo BC. In an analytic population-based study of $\mathrm{BC}$ after radiation for $\mathrm{HL}$, archived paraffin-embedded tissues from 19 women were compared with de novo BC. ${ }^{41} \mathrm{BC}$ after HL was characterized by a 4.2 -fold increase $(P=.16)$ in microsatellite alterations, reflecting widespread genomic instability. ${ }^{41}$ Moreover, loss of heterozygosity in several chromosomes were significantly greater among HL survivors versus those with de novo $\mathrm{BC} .{ }^{41}$ In another series, $\mathrm{BC}$ after $\mathrm{HL}$, as compared with de novo $\mathrm{BC}$, had a gene expression profile characterized by high proliferation, more aggressive tumor type (in approximately $80 \%$ of HL survivors), ${ }^{42}$ and greater likelihood of chromosomal aberrations (unpublished work discussed in Broeks et $\mathrm{al}^{42}$ ). Another investigation showed no differences in germ-line mutations in the ataxia-telangiectasia gene between $\mathrm{BC}$ after $\mathrm{HL}$ and de novo BC. ${ }^{43}$

Because of the long follow-up in our study, we are able to report for the first time 15-year OS of HL survivors who develop BC. Two matched-pair analyses have also compared OS of HL survivors against women with a first or only $\mathrm{BC},{ }^{20,21}$ although with far fewer patients and less follow-up than in our study. One series analyzed $21 \mathrm{HL}$ survivors and another evaluated 53 patients (of whom 35 patients had $\mathrm{HL})$. In neither study was OS significantly different between HL survivors and those with de novo $\mathrm{BC}$ at time points of 5 to 10 years. ${ }^{20,21}$

Compared with women with first or only BC, HL survivors were relatively younger at time of $\mathrm{BC}$ diagnosis; diagnosed in relatively later decades, in part reflecting the typical latency period of $\geq 10$ years required for radiation-associated solid tumor ${ }^{27}$; and had a higher sociodemographic status, which is known to be correlated with $\mathrm{HL}^{44,45}$ BCs among HL survivors were significantly more likely to be characterized by pathologic features of poorer grade, ER negativity, and PR negativity. Whether the greater risk of ER- and PR-negative tumors in our study may somehow reflect chemotherapy-induced (specifically mechlorethamine, vincristine, procarbazine, and pred- nisone) ovarian ablation ${ }^{46,47}$ is not known. Yahalom et al ${ }^{17}$ compared the histologic features of $45 \mathrm{BCs}$ in 37 patients previously treated for HL with those in 935 patients with first BC. Nuclear grade, histologic grade, lymphocytic reaction, and lymphatic invasion were similar between the two groups.

The primary strength of the current study is the large number of patients (> 400,000) including 298 with BC after HL. All patients were derived from population-based registries, with no selection biases and with long-term follow-up, strengthening the generalizability of our results. Weaknesses of the SEER database include lack of information about radiation dose and fields as well as whether or not patients received chemotherapy (or what agents they received), factors important in predicting BC risks in patients with HL. ${ }^{5,9,12,16}$ In addition, it should be recognized that even the initial course of radiotherapy is underreported in the SEER program. ${ }^{48}$ With respect to BC-CSS, the power to detect covariate effects was limited because only 52 patients with HL-BC died as a result of $\mathrm{BC}$, the most common cause of death in these patients. There were also small numbers of deaths from other causes.

Nonetheless, our results underscore the importance of continuing to monitor HL survivors who develop BC for late cardiac complications $^{23}$ and additional malignancies, as well as the need to counsel patients on preventive measures such as smoking cessation and healthy lifestyle modifications. Systematic lifelong follow-up is needed to examine the emergence of long-term toxicity and associated morbidity and mortality. ${ }^{49,50}$ Future research should be directed at examining the underlying cancer biology and etiology of treatmentinduced cancers, as well as inherent and treatment-induced genetic susceptibility of HL survivors. Genetic susceptibility may not only impact risk of developing additional cancers, but also subsequent survival, and warrants careful investigation.

\section{AUTHORS' DISCLOSURES OF POTENTIAL CONFLICTS} OF INTEREST

The author(s) indicated no potential conflicts of interest.

\section{AUTHOR CONTRIBUTIONS}

Conception and design: Michael T. Milano, Lois B. Travis

Collection and assembly of data: Michael T. Milano, Huilin Li, Mitchell

H. Gail, Lois B. Travis

Data analysis and interpretation: Michael T. Milano, Huilin Li, Mitchell H. Gail, Louis S. Constine, Lois B. Travis

Manuscript writing: Michael T. Milano, Lois B. Travers

Final approval of manuscript: Michael T. Milano, Huilin Li, Mitchell H. Gail, Louis S. Constine, Lois B. Travers

\section{REFERENCES}

1. Donaldson SS, Hancock SL, Hoppe RT: The Janeway lecture: Hodgkin's disease-Finding the balance between cure and late effects. Cancer J Sci Am 5:325-333, 1999

2. Dores GM, Metayer C, Curtis RE, et al: Second malignant neoplasms among long-term survivors of Hodgkin's disease: A population-based evaluation over 25 years. J Clin Oncol 20:3484-3494, 2002

3. Aleman BM, van den Belt-Dusebout AW, Klokman WJ, et al: Long-term cause-specific mor- tality of patients treated for Hodgkin's disease. J Clin Oncol 21:3431-3439, 2003

4. van Leeuwen FE, Klokman WJ, Veer MB, et al: Long-term risk of second malignancy in survivors of Hodgkin's disease treated during adolescence or young adulthood. J Clin Oncol 18:487-497, 2000

5. Franklin JG, Paus MD, Pluetschow A, et al: Chemotherapy, radiotherapy and combined modality for Hodgkin's disease, with emphasis on second cancer risk. Cochrane Database Syst Rev 4:CD003187, 2005

6. Mauch PM, Kalish LA, Marcus KC, et al: Long-term survival in Hodgkin's disease relative impact of mortality, second tumors, infection, and cardiovascular disease. Cancer J Sci Am 1:33-42, 1995

7. Ng AK, Bernardo MP, Weller $E$, et al: Long-term survival and competing causes of death in patients with early-stage Hodgkin's disease treated at age 50 or younger. J Clin Oncol 20:2101-2108, 2002

8. Hodgson DC: Hodgkin lymphoma: The follow-up of long-term survivors. Hematol Oncol Clin North Am 22:233-244, vi, 2008

9. Hancock SL, Tucker MA, Hoppe RT: Breast cancer after treatment of Hodgkin's disease. J Natl Cancer Inst 85:25-31, 1993

10. Aisenberg $A C$, Finkelstein DM, Doppke KP, et al: High risk of breast carcinoma after irradiation of 
young women with Hodgkin's disease. Cancer 79 1203-1210, 1997

11. Wendland MM, Tsodikov A, Glenn MJ, et al: Time interval to the development of breast carcinoma after treatment for Hodgkin disease. Cancer 101:1275-1282, 2004

12. Travis LB, Hill D, Dores GM, et al: Cumulative absolute breast cancer risk for young women treated for Hodgkin lymphoma. J Natl Cancer Inst 97:1428-1437, 2005

13. Basu SK, Schwartz C, Fisher SG, et al: Unilateral and bilateral breast cancer in women surviving pediatric Hodgkin's disease. Int J Radiat Oncol Bio Phys 72:34-40, 2008

14. Alm El-Din MA, Hughes KS, Finkelstein DM, et al: Breast cancer after treatment of Hodgkin's lymphoma: Risk factors that really matter. Int $J$ Radiat Oncol Biol Phys 73:69-74, 2009

15. Cutuli B, Borel C, Dhermain F, et al: Breast cancer occurred after treatment for Hodgkin's disease: Analysis of 133 cases. Radiother Oncol 59 247-255, 2001

16. De Bruin ML, Sparidans J, Van't Veer MB et al: Breast cancer risk in female survivors of Hodgkin's lymphoma: Lower risk after smaller radiation volumes. J Clin Oncol 27:4239-4246, 2009

17. Yahalom J, Petrek JA, Biddinger PW, et al: Breast cancer in patients irradiated for Hodgkin's disease: A clinical and pathologic analysis of 45 events in 37 patients. J Clin Oncol 10:1674-1681, 1992

18. Wolden SL, Hancock SL, Carlson RW, et al: Management of breast cancer after Hodgkin's disease. J Clin Oncol 18:765-772, 2000

19. Cutuli B, Dhermain F, Borel $C$, et al: Breast cancer in patients treated for Hodgkin's disease: Clinical and pathological analysis of 76 cases in 63 patients. Eur J Cancer 33:2315-2320, 1997

20. Sanna G, Lorizzo K, Rotmensz N, et al: Breast cancer in Hodgkin's disease and non-Hodgkin's lymphoma survivors. Ann Oncol 18:288-292, 2007

21. Alm El-Din $M$, Hughes $K S$, Raad RA, et al: Clinical outcome of breast cancer occurring after treatment for Hodgkin's lymphoma: Case-control analysis. Radiat Oncol 4:19, 2009

22. Ng AK, Bernardo MV, Weller E, et al: Second malignancy after Hodgkin disease treated with radiation therapy with or without chemotherapy: Longterm risks and risk factors. Blood 100:1989-1996, 2002

23. Adams MJ, Lipshultz SE, Schwartz C, et al: Radiation-associated cardiovascular disease: Manifestations and management. Semin Radiat Oncol 13:346-356, 2003

24. Heidenreich PA, Schnittger I, Strauss HW, et al: Screening for coronary artery disease after me- diastinal irradiation for Hodgkin's disease. J Clin Oncol 25:43-49, 2007

25. Surveillance, Epidemiology, and End Results (SEER) Program: SEER*Stat Database: IncidenceSEER 9 Regs Limited-Use, November 2008 Sub (1973-2006) (Katrina/Rita Population Adjustment). Linked To County Attributes: Total US, 1969-2006 Counties. Bethesda, MD, National Cancer Institute, Division of Cancer Control and Population Sciences, Surveillance Research Program, Cancer Statistics Branch, released April 2009, based on the November 2008 submission. www.seer.cancer.gov

26. $R$ Development Core Team: R: A Language and Environment for Statistical Computing, reference index version 2.7.0. Vienna, Austria, R Foundation for Statistical Computing, 2005

27. Boice JD: Ionizing radiation, in Schottenfeld D, Fraumeni JF (eds): Cancer Epidemiology and Prevention. New York, NY, Oxford University Press, 2006, pp 259-293

28. Hoffman KE, Chen MH, Punglia RS, et al: Influence of year of diagnosis, patient age, and sociodemographic status on recommending adjuvant radiation treatment for stage I testicular seminoma. J Clin Oncol 26:3937-3942, 2008

29. Salloum E, Doria R, Schubert W, et al: Second solid tumors in patients with Hodgkin's disease cured after radiation or chemotherapy plus adjuvant low-dose radiation. J Clin Oncol 14:2435-2443, 1996

30. Hodgson DC, Gilbert ES, Dores GM, et al: Long-term solid cancer risk among 5-year survivors of Hodgkin's lymphoma. J Clin Oncol 25:1489-1497, 2007

31. van Leeuwen FE, Klokman WJ, Hagenbeek A, et al: Second cancer risk following Hodgkin's disease: A 20-year follow-up study. J Clin Oncol 12: 312-325, 1994

32. Rueffer $U$, Josting $A$, Franklin J, et al: NonHodgkin's lymphoma after primary Hodgkin's disease in the German Hodgkin's Lymphoma Study Group: Incidence, treatment, and prognosis. J Clin Oncol 19:2026-2032, 2001

33. Travis LB, Gospodarowicz M, Curtis RE, et al: Lung cancer following chemotherapy and radiotherapy for Hodgkin's disease. J Natl Cancer Inst 94: 182-192, 2002

34. Gilbert ES, Stovall M, Gospodarowicz M, et al: Lung cancer after treatment for Hodgkin's disease: Focus on radiation effects. Radiat Res 159:161-173, 2003

35. Kaufman EL, Jacobson JS, Hershman DL, et al: Effect of breast cancer radiotherapy and cigarette smoking on risk of second primary lung cancer. J Clin Oncol 26:392-398, 2008

36. Prochazka M, Hall P, Gagliardi G, et al: Ionizing radiation and tobacco use increases the risk of a subsequent lung carcinoma in women with breast cancer: Case-only design. J Clin Oncol 23:74677474, 2005

37. Tennis $M$, Singh $B$, Hjerpe $A$, et al: Patholog ical confirmation of primary lung cancer following breast cancer. Lung Cancer 69:40-45, 2010

38. Bird BR, Swain SM: Cardiac toxicity in breast cancer survivors: Review of potential cardiac problems. Clin Cancer Res 14:14-24, 2008

39. Gagliardi G, Constine LS, Moiseenko V et al: Radiation-associated heart injury. Int J Radiat Oncol Biol Phys 76:S77-85, 2010

40. Schonfeld SJ, Gilbert ES, Dores GM, et al: Acute myeloid leukemia following Hodgkin lymphoma: A population-based study of 35,511 patients. J Natl Cancer Inst 98:215-218, 2006

41. Behrens C, Travis LB, Wistuba II, et al: Molecular changes in second primary lung and breast cancers after therapy for Hodgkin's disease. Cancer Epidemiol Biomarkers Prev 9:1027-1035, 2000

42. Broeks A, Braaf LM, Wessels LFA, et al: Radiation-associated breast tumors display a distinct gene expression profile. Int J Radiat Oncol Biol Phys 76:540-547, 2010

43. Broeks A, Russell NS, Floore AN, et al: Increased risk of breast cancer following irradiation for Hodgkin's disease is not a result of ATM germline mutations. Int J Radiat Biol 76:693-698, 2000

44. Glaser SL: Regional variation in Hodgkin's disease incidence by histologic subtype in the US Cancer 60:2841-2847, 1987

45. Mueller NE, Grufferman S: Hodgkin lymphoma, in Schottenfeld D, Fraumeni JF (eds): Cancer Epidemiology and Prevention. New York, NY Oxford University Press, 2006, pp 872-897

46. Horning SJ, Hoppe RT, Kaplan HS, et al: Female reproductive potential after treatment for Hodgkin's disease. N Engl J Med 304:1377-1382, 1981

47. Travis LB, Hill DA, Dores GM, et al: Breast cancer following radiotherapy and chemotherapy among young women with Hodgkin disease. JAMA 290:465-475, 2003

48. Curtis RE, Ries LG: Methods, in Curtis RE, Freedman DM, Ron E, et al (eds): New Malignancies Among Cancer Survivors: SEER Cancer Registries, 1973-2000. Bethesda, MD, National Cancer Institute, NIH publication 05-5302, 2006

49. Hudson MM, Mertens AC, Yasui $Y$, et al: Health status of adult long-term survivors of childhood cancer: A report from the Childhood Cancer Survivor Study. JAMA 290:1583-1592, 2003

50. Children's Oncology Group: Long-term follow-up guidelines for survivors of childhood, adolescent and young adult cancers, Version 3.0. Arcadia, CA, Children's Oncology Group, October 2008. www.survivorshipguidelines.org 